\title{
Coherence revival during the attosecond electronic and nuclear quantum photodynamics of the ozone molecule
}

\author{
G. J. Halász, ${ }^{1}$ A. Perveaux,${ }^{2}$ B. Lasorne,${ }^{2}$ M. A. Robb,${ }^{3}$ F. Gatti, ${ }^{2}$ and Á. Vibók ${ }^{4, *}$ \\ ${ }^{1}$ Department of Information Technology, University of Debrecen, P.O. Box 12, H-4010 Debrecen, Hungary \\ ${ }^{2}$ CTMM, Institut Charles Gerhardt Montpellier, Université Montpellier 2, F-34095 Montpellier, France \\ ${ }^{3}$ Department of Chemistry, Imperial College London, London SW7 2AZ, U.K. \\ ${ }^{4}$ Department of Theoretical Physics, University of Debrecen, P.O. Box 5, H-4010 Debrecen, Hungary
}

(Received 22 May 2013; published 27 August 2013)

\begin{abstract}
A coherent superposition of two electronic states of ozone (ground and Hartley $B$ ) is prepared with a UV pump pulse. Using the multiconfiguration time-dependent Hartree approach, we calculate the subsequent time evolution of the two corresponding nuclear wave packets and the coherence between them. The resulting wave packet shows an oscillation between the two chemical bonds. Even more interesting, the coherence between the two electronics states reappears after the laser pulse is switched off, which could be observed experimentally with an attosecond probe pulse.
\end{abstract}

DOI: 10.1103/PhysRevA.88.023425

PACS number(s): 33.80.Wz, 31.15.xv

\section{INTRODUCTION}

The construction of single few-cycle ultrashort laser pulses or trains of ultrashort pulses enables the control of different photophysical and photochemical processes. Experimentalists can excite and probe electron dynamics in atoms and molecules in real time [1-10], monitoring the subfemtosecond motion of valence electrons over a multifemtosecond time span that results in taking real-time snapshots of ultrafast transformations of matter. Successful theoretical and experimental investigations of the electron dynamics of the $\mathrm{Kr}$ atom have been performed recently $[7,8,10]$. However, extending these techniques to molecules remains a challenge. Problems arise because electron dynamics in molecules often are strongly coupled to nuclear dynamics.

For molecules, various approaches have been developed so far. In most attophysics simulations, only the electron dynamics is treated, and the molecular geometries (nuclear positions) are assumed to be fixed [11-16]. Within this approach an arbitrarily large molecule can be examined. To achieve this, one needs to use an ultrashort laser pulse during the probe process. If longer probe laser pulses are applied, the nuclei have time to move. In this situation the nuclear dynamics has to be considered as well. For the simplest ion, $\mathrm{H}_{2}^{+}$, or molecule, $\mathrm{H}_{2}$, it is easily feasible [20-26], but for diatomics containing many electrons or even for polyatomics the problem to be solved is more complex and difficult [27-29]. In the first situation (e.g., $\mathrm{H}_{2}^{+}$or $\mathrm{H}_{2}$ ) the total time-dependent Schrödinger equation can be solved numerically including explicitly both the electronic and the nuclear degrees of freedom. In contrast, the case of many electrons or polyatomics implies facing either the problem of electron correlation or that of a large number of nuclear degrees of freedom [19].

Recently, we proposed a nonadiabatic scheme for description of the coupled electron and nuclear motion in the ozone molecule [30]. An initial coherent nonstationary state was prepared by two pump pulses. It was a superposition of different weakly bound states in the Chappuis band [38]

\footnotetext{
*vibok@phys.unideb.hu
}

(which are populated by NIR radiation), as well as in the Hartley band [38] (which is populated by the third harmonic pulse). In this situation neither the electrons nor the nuclei were in a stationary state, and we used nonadiabatic quantum dynamics simulations. As the transition dipole moments are very different in the ground and Hartley states compared to the ground and Chappuis bands, we had to apply significantly different intensities for the two pump pulses not to obtain differences in the population of the Hartley versus the Chappuis states larger than one order of magnitude. Consequently, we used $2 \times 10^{11}$ and $10^{14} \mathrm{~W} / \mathrm{cm}^{2}$ intensities to populate the Hartley and Chappuis states, respectively, which is not trivial to achieve experimentally, while further probing the system with an attosecond XUV pulse.

However, opportunities arise to reasonably simplify the task. As we excite only the $B$ state of the Hartley band with a much higher intensity pump pulse than in our previous work, the population obtained in this state is more pronounced. The nonstationary state is a coherent superposition of these two (ground and $B$ ) electronic states, and the motion of the electronic wave packet can thus be probed assuming much less complicated experimental setups than in the previous situation.

Our original motivation was to perform a numerical simulation for an experimentally easier situation. An interesting phenomenon emerged from this investigation: the revival of the electronic coherence after the pump pulse is off, which could also be probed experimentally. The main aim of the present paper is to report this uncommon finding, which can be explained because we only coupled the $X$ and $B$ electronic states, between which there is no nonadiabatic coupling and no conical intersection.

As in our previous work, the nuclear wave packets, the electronic populations, the relative electronic coherence between the ground $X$ and the $B$ electronic states, and the electron wave packet dynamics were calculated. The time evolution of the electronic motion was plotted in the FranckCondon (FC) region only due to the localization of the nuclear wave packet around this point during the first 5-6 fs. The electron density shows a fast oscillation between both chemical bonds. This should reflect a time-dependent anisotropy pattern in time-resolved photoelectron angular distributions obtained 
experimentally by means of attosecond XUV probe pulses $[17,18]$.

The paper is organized as follows. Section II gives some insights into the formalism and methods used here. Results and their discussion are presented in Sec. III. Section IV is devoted to our conclusions. Some useful remarks about the electronic-structure results are provided in the Appendix.

\section{METHODS AND FORMALISM}

In this section a short summary of the methods and formalism used in our simulations is given. For more details we refer to our former paper [30].

\section{A. Time-dependent molecular Schrödinger equation}

The adiabatic partition formalism (beyond BornOppenheimer [31]) assumes the total molecular wave function $\Psi_{\mathrm{tot}}\left(\vec{r}_{\mathrm{el}}, \vec{R}, t\right)$ as a sum of the products of electronic wave functions, $\psi_{\mathrm{el}}^{k}\left(\vec{r}_{\mathrm{el}} ; \vec{R}\right)$, and nuclear wave packets, $\Psi_{\mathrm{nuc}}^{k}(\vec{R}, t)$ :

$$
\Psi_{\text {tot }}\left(\vec{r}_{\mathrm{el}}, \vec{R}, t\right)=\sum_{k=1}^{n} \Psi_{\text {nuc }}^{k}(\vec{R}, t) \psi_{\mathrm{el}}^{k}\left(\vec{r}_{\mathrm{el}} ; \vec{R}\right) .
$$

Here $k$ denotes the $k$ th adiabatic electronic state, and $\vec{r}_{\mathrm{el}}$ and $\vec{R}$ are the electronic and the nuclear coordinates, respectively. We are interested in solving the coupled evolution of the nuclear wave packets, $\Psi_{\text {nuc }}^{k}(\vec{R}, t)$, by inserting the product ansatz, (1), into the time-dependent Schrödinger equation of the full molecular Hamiltonian. Integrating over the electronic coordinates one obtains the coupled nuclear Schrödinger equations:

$$
i \hbar \frac{\partial}{\partial t} \Psi_{\text {nuc }}^{k}(\vec{R}, t)=\sum_{l=1, n} H_{k, l} \Psi_{\text {nuc }}^{l}(\vec{R}, t) .
$$

Here $H_{k, l}$ is the matrix element of the vibronic Hamiltonian, which reads, e.g., for $n=2$,

$$
H=\left(\begin{array}{cc}
T_{\mathrm{nuc}}+V_{k} & K_{k, l} \\
-K_{k, l} & T_{\mathrm{nuc}}+V_{l}
\end{array}\right),
$$

where $T_{\text {nuc }}$ is the nuclear kinetic energy, $V_{k}(k=1, \ldots, n)$ is the $k$ th adiabatic potential energy, and $K_{k, l}$ with $k \neq l$ is the vibronic coupling term between the $(k, l)$ th electronic states. The latter contains the nonadiabatic coupling term. In the presence of an external electric field the light-matter interaction, $-\vec{\mu}(k, l) \cdot \vec{E}(t)$ (electric dipole approximation), where $\vec{E}(t)$ is an external field resonant between the $k$ th and the $l$ th states and $\vec{\mu}(k, l)$ is the $\vec{R}$-dependent transition dipole moment, is also included in this coupling term. In the present situation, there is no significant nonadiabatic coupling between the ground and the Hartley state, therefore $K_{k, l}$ denotes only the light-matter interaction.

One has to solve the time-dependent nuclear Schrödinger equation given by Eq. (2). One of the most efficient approaches for this is the MCTDH (multiconfiguration time-dependent Hartree) method [32-35].

The MCTDH nuclear wave packets, $\Psi_{\text {nuc }}^{k}(\vec{R}, t)$, contain all the information about the relative phases between the electronic states. Therefore $\Psi_{\text {nuc }}^{k}(\vec{R}, t)$ can also be written as

$$
\Psi_{\mathrm{nuc}}^{k}(\vec{R}, t)=\exp \left[-i W_{k}(\vec{R}) t / \hbar\right] a_{k}(\vec{R}, t) .
$$

Here, $W_{k}(\vec{R})$ is the potential energy of the $k$ th state. The first part of this wave function is the phase factor, $\exp \left[-i W_{k}(\vec{R}) t / \hbar\right]$, of the $k$ th state, which oscillates very rapidly.

\section{B. Density matrix}

Here we define the working formulas that are used in the next section. Calculating them requires only the knowledge of the nuclear wave packets.

The two-dimensional nuclear density function (depending on $R_{1}$ and $R_{2}$, the two bond lengths, and integrated over $\theta$, the bond angle) is

$$
\begin{aligned}
\left|\Psi_{\mathrm{nuc}}^{i}\left(R_{1}, R_{2}, t\right)\right|^{2}= & \int \Psi_{\mathrm{nuc}}^{i}\left(R_{1}, R_{2}, \theta, t\right) \Psi_{\mathrm{nuc}}^{i *}\left(R_{1}, R_{2}, \theta, t\right) \\
& \times \sin \theta d \theta
\end{aligned}
$$

The total density matrix of the molecule is defined as

$$
\begin{aligned}
\rho_{i i^{\prime}}\left(\vec{R}, \vec{R}^{\prime}, t\right)= & \left\langle\psi_{\mathrm{el}}^{i}\left(\vec{r}_{\mathrm{el}} ; \vec{R}\right) \mid \Psi_{\mathrm{tot}}\left(\vec{r}_{\mathrm{el}}, \vec{R}, t\right)\right\rangle \\
& \times\left\langle\Psi_{\mathrm{tot}}\left(\vec{r}_{\mathrm{el}}, \vec{R}^{\prime}, t\right) \mid \psi_{\mathrm{el}}^{i^{\prime}}\left(\vec{r}_{\mathrm{el}} ; \vec{R}^{\prime}\right)\right\rangle \\
= & \Psi_{\text {nuc }}^{i}(\vec{R}, t) \Psi_{\text {nuc }}^{i^{\prime} *}\left(\vec{R}^{\prime}, t\right),
\end{aligned}
$$

where angle brackets denote integration over the electronic coordinates only.

The electronic population function of the $i$ th state is

$$
P_{i}(t)=\int \rho_{i i}(\vec{R}, \vec{R}, t) d \vec{R}
$$

Analogously, we define the electronic relative coherence between the $i$ th and the $i^{\prime}$ th electronic states as

$$
C_{i i^{\prime}}(t)=\int \rho_{i i^{\prime}}(\vec{R}, \vec{R}, t) d \vec{R} / \sqrt{P_{i}(t) P_{i^{\prime}}(t)} .
$$

\section{Electronic structure treatment}

Here we briefly review the represention used for the electronic wave packet. We consider only two (ground and Hartley B) electronic states. At the FC geometry, each electronic state can be represented by its charge density in the three-dimensional space,

$$
\begin{aligned}
\rho^{i}\left(\vec{r}, \vec{R}_{\mathrm{FC}}\right)= & N \int_{N(\text { spin })} d \sigma_{1} d \sigma_{2} \ldots d \sigma_{N} \int_{N-1(\text { space })} d \tau_{2} \ldots d \tau_{N} \\
& \times\left|\psi_{\mathrm{el}}^{i}\left(\vec{r}_{1}=\vec{r}, \sigma_{1}, \vec{r}_{2}, \sigma_{2}, \ldots, \vec{r}_{N}, \sigma_{N} ; \vec{R}_{\mathrm{FC}}\right)\right|^{2} .
\end{aligned}
$$

Here $i=X$ or $B$. It is often called the one-electron density, although rigorously, it is $N$ times the one-electron density summed over both spin states of electron 1. It is defined as the density of probability of finding 1 among $N$ electrons in any spin state (up or down) at point $\vec{r} \equiv(x, y, z)$ and time $t$ for the molecule in states $X$ and $B$, respectively, and geometry $\vec{R}_{\mathrm{FC}}$. 
The transition density between state $X$ and state $B$ is defined in the three-dimensional space as

$$
\begin{aligned}
\gamma^{X B}\left(\vec{r} ; \vec{R}_{\mathrm{FC}}\right)= & N \int_{N(\text { spin })} d \sigma_{1} d \sigma_{2} \ldots d \sigma_{N} \int_{N-1(\text { space })} d \tau_{2} \ldots d \tau_{N} \\
& \times \psi_{\mathrm{el}}^{X *}\left(\vec{r}_{1}=\vec{r}, \sigma_{1}, \vec{r}_{2}, \sigma_{2}, \ldots, \vec{r}_{N}, \sigma_{N} ; \vec{R}_{\mathrm{FC}}\right) \\
& \times \psi_{\mathrm{el}}^{B}\left(\vec{r}_{1}=\vec{r}, \sigma_{1}, \vec{r}_{2}, \sigma_{2}, \ldots, \vec{r}_{N}, \sigma_{N} ; \vec{R}_{\mathrm{FC}}\right) .
\end{aligned}
$$

It is a measure of the interference between both states. The total molecular wave packet observed at a fixed geometry, here at the FC point, is a coherent mixture of both electronic states, whereby the time-dependent coefficients are the nuclear wave packets at the FC point:

$$
\begin{aligned}
\Psi_{\mathrm{mol}} & \left(\vec{r}_{1}, \sigma_{1}, \vec{r}_{2}, \sigma_{2}, \ldots, \vec{r}_{N}, \sigma_{N} ; \vec{R}_{\mathrm{FC}}, t\right) \\
= & \Psi_{\mathrm{nuc}}^{X}\left(\vec{R}_{\mathrm{FC}}, t\right) \psi_{\mathrm{el}}^{X}\left(\vec{r}_{1}, \sigma_{1}, \vec{r}_{2}, \sigma_{2}, \ldots, \vec{r}_{N}, \sigma_{N} ; \vec{R}_{\mathrm{FC}}\right) \\
& +\Psi_{\mathrm{nuc}}^{B}\left(\vec{R}_{\mathrm{FC}}, t\right) \psi_{\mathrm{el}}^{B}\left(\vec{r}_{1}, \sigma_{1}, \vec{r}_{2}, \sigma_{2}, \ldots, \vec{r}_{N}, \sigma_{N} ; \vec{R}_{\mathrm{FC}}\right)
\end{aligned}
$$

Thus, the corresponding total time-dependent charge density reads

$$
\begin{aligned}
\rho^{\mathrm{tot}}\left(\vec{r}, t ; \vec{R}_{\mathrm{FC}}\right)= & \left|\Psi_{\mathrm{nuc}}^{X}\left(\vec{R}_{\mathrm{FC}}, t\right)\right|^{2} \rho^{X}\left(\vec{r} ; \vec{R}_{\mathrm{FC}}\right) \\
& +\left|\Psi_{\mathrm{nuc}}^{B}\left(\vec{R}_{\mathrm{FC}}, t\right)\right|^{2} \rho^{B}\left(\vec{r} ; \vec{R}_{\mathrm{FC}}\right) \\
& +2 \operatorname{Re} \Psi_{\mathrm{nuc}}^{X *}\left(\vec{R}_{\mathrm{FC}}, t\right) \Psi_{\mathrm{nuc}}^{B}\left(\vec{R}_{\mathrm{FC}}, t\right) \gamma^{X B}\left(\vec{r} ; \vec{R}_{\mathrm{FC}}\right) .
\end{aligned}
$$

The latter quantity depends on time, but the difference from the ground-state charge density is difficult to notice because most electrons are unaffected by the electronic transition to state $B$. To get better contrast, we can represent a differential charge density. If we assume that the effect of the coupling with the laser pump pulse only affects the electrons for the duration of the observation, then there is no transfer of local population density from $\vec{R}_{\mathrm{FC}}$ to other values of $\vec{R}$. As long as this approximation holds, then

$$
\begin{aligned}
\left|\Psi_{\mathrm{nuc}}^{X}\left(\vec{R}_{\mathrm{FC}}, t<0\right)\right|^{2}= & \left|\Psi_{\mathrm{nuc}}^{B}\left(\vec{R}_{\mathrm{FC}}, t>0\right)\right|^{2} \\
& +\left|\Psi_{\mathrm{nuc}}^{X}\left(\vec{R}_{\mathrm{FC}}, t>0\right)\right|^{2},
\end{aligned}
$$

where the pulse is switched on at $t=0$, and the corresponding change in charge density due to the pulse reads

$$
\Delta \rho^{B}\left(\vec{r}, t>0 ; \vec{R}_{\mathrm{FC}}\right)=\rho^{\text {tot }}\left(\vec{r}, t>0 ; \vec{R}_{\mathrm{FC}}\right)-\rho^{\mathrm{tot}}\left(\vec{r}, t<0 ; \vec{R}_{\mathrm{FC}}\right) .
$$

An estimate of the excited-state differential charge density at the FC point can thus be defined as

$$
\begin{aligned}
\Delta \rho^{B}\left(\vec{r}, t ; \vec{R}_{\mathrm{FC}}\right)= & \rho^{\text {tot }}\left(\vec{r}, t ; \vec{R}_{\mathrm{FC}}\right)-\left[\left|\Psi_{\mathrm{nuc}}^{X}\left(\vec{R}_{\mathrm{FC}}, t\right)\right|^{2}\right. \\
& \left.+\left|\Psi_{\mathrm{nuc}}^{B}\left(\vec{R}_{\mathrm{FC}}, t\right)\right|^{2}\right] \rho^{X}\left(\vec{r} ; \vec{R}_{\mathrm{FC}}\right) \\
= & \left|\Psi_{\mathrm{nuc}}^{B}\left(\vec{R}_{\mathrm{FC}}, t\right)\right|^{2}\left[\rho^{B}\left(\vec{r} ; \vec{R}_{\mathrm{FC}}\right)-\rho^{X}\left(\vec{r} ; \vec{R}_{\mathrm{FC}}\right)\right] \\
& +2 \operatorname{Re} \Psi_{\mathrm{nuc}}^{X *}\left(\vec{R}_{\mathrm{FC}}, t\right) \Psi_{\mathrm{nuc}}^{B}\left(\vec{R}_{\mathrm{FC}}, t\right) \gamma^{X B}\left(\vec{r} ; \vec{R}_{\mathrm{FC}}\right) \\
= & \left|\Psi_{\mathrm{nuc}}^{B}\left(\vec{R}_{\mathrm{FC}}, t\right)\right|^{2} \triangle \rho^{B}\left(\vec{r} ; \vec{R}_{\mathrm{FC}}\right) \\
& +2 \operatorname{Re} \Psi_{\mathrm{nuc}}^{X *}\left(\vec{R}_{\mathrm{FC}}, t\right) \Psi_{\mathrm{nuc}}^{B}\left(\vec{R}_{\mathrm{FC}}, t\right) \gamma^{X B}\left(\vec{r} ; \vec{R}_{\mathrm{FC}}\right),
\end{aligned}
$$

where $\triangle \rho^{B}\left(\vec{r} ; \vec{R}_{\mathrm{FC}}\right)=\rho^{B}\left(\vec{r} ; \vec{R}_{\mathrm{FC}}\right)-\rho^{X}\left(\vec{r} ; \vec{R}_{\mathrm{FC}}\right)$.

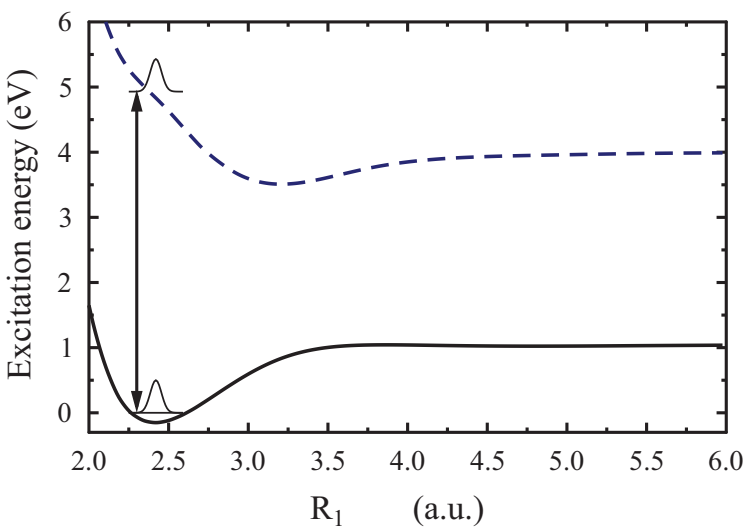

FIG. 1. (Color online) Potential energy surfaces of ozone as functions of the dissociation coordinate: ground state $(X$; solid line) and Hartley state ( $B$; dashed line). The arrow denotes excitation of the $B$ state. The other two coordinates are fixed at $R_{2}=2.43$ a.u. and the bond angle is $\theta=117^{\circ}$.

\section{RESULTS AND DISCUSSION}

In the present work only two electronic states of ozone are involved in the numerical simulations: the ground state $X$, with ${ }^{1} A_{1}$ symmetry;and the highly excited $B$ state in the Hartley band, with ${ }^{1} B_{2}$ symmetry. In Fig. 1 we show a one-dimensional cut along the $\mathrm{O}-\mathrm{O}$ bond through the potential energy surfaces (PESs) of both electronic states. We note here, as there is no nonadiabatic coupling between these two states, that the adiabatic and diabatic energies are identical. A UV linearly polarized Gaussian laser pump pulse was used to prepare a coherent superposition of the two stationary electronic states: the ground $X$ and the populated $B$ states. The center wavelength and the intensity of the pulse are $260 \mathrm{~nm}$ and $10^{13} \mathrm{~W} / \mathrm{cm}^{2}$, respectively. The FWHM is 3 fs. The PESs and $\vec{R}$-dependent
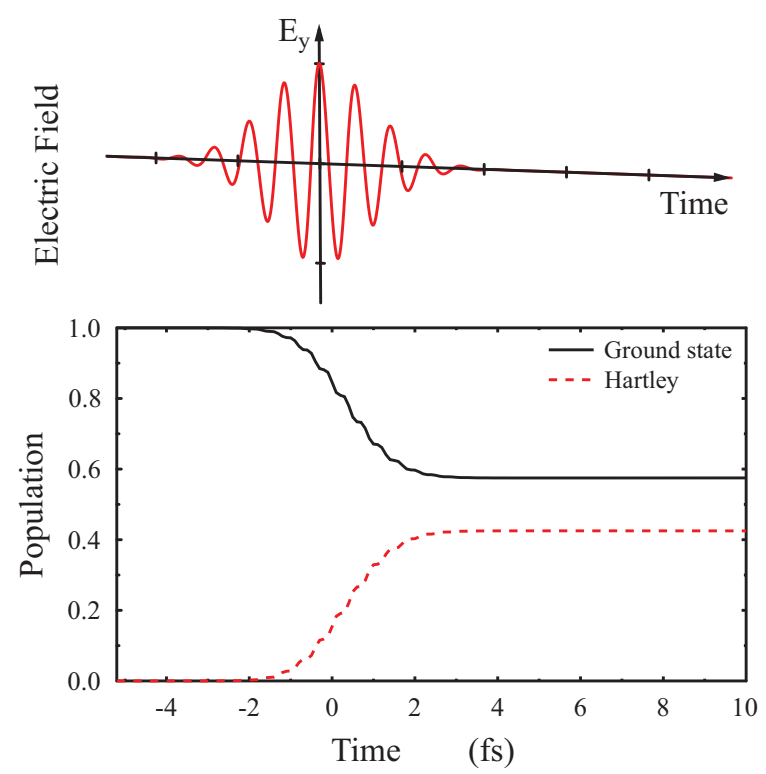

FIG. 2. (Color online) Top: Applied electric field. Bottom: Time evolution of the diabatic populations in the ground $(X)$ and diabatic excited $(B)$ states. 


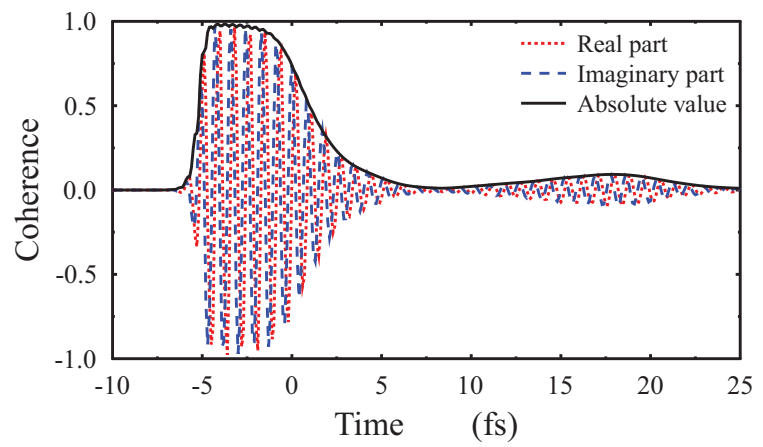

FIG. 3. (Color online) Relative electronic coherence as a function of time. The real part, the imaginary part, and the absolute value of the relative electronic coherence between the ground $(X)$ and the Hartley (B) states.

dipole moments occurring in the radiative coupling terms were taken from Refs. [36], [38], and [39].

The FC point has $C_{2 v}$ symmetry. As a consequence, only the $y$ component $\left(B_{2}\right)$ of the transition dipole between the ground state $X\left({ }^{1} A_{1}\right)$ and the Hartley $B$ state $\left({ }^{1} B_{2}\right)$ is nonzero. Therefore the only effective polarization of the electric field is $y$ (seeFig. 2, top).

In the lower panel in Fig. 2 the total populations versus time [see Eq. (7)] are displayed in the ground and Hartley $B$ states up to $t=10 \mathrm{fs}$ (note that they stay constant up to the end of the simulation, at $t=35 \mathrm{fs}$ ). The Hartley $B$ state absorbs very strongly due to the large value of the transition dipole moment with the ground state [37]. In the $(-2,2)$ fs interval the population grows continuously, then reaches its maximum and remains at this value throughout the studied time period. The $B$ state is populated with a yield of about $40 \%$. The laser intensity $\left(10^{13} \mathrm{~W} / \mathrm{cm}^{2}\right)$ is thus high enough to transfer nearly half the amount of the wave packet from the ground state to the $B$ state.

Figure 3 shows the electronic relative coherence, Eq. (8), between the ground and the $B$ states. In the first time period the coherence increases very rapidly and reaches its maximum. It retains this value for 3-4 fs, which is approximately equivalent to the duration of the laser pulse, and then it decays during the next 6-7 fs. However, this is not the end of the process: a few femtoseconds later $(\sim 5 \mathrm{fs})$, the coherence reappears in contrast with what was observed in Ref. [30]. This revival of coherence proves that we have created, to some extent, a "true" coherent superposition, in that it is not forced by the presence of an external field. This phenomenon could certainly be enhanced experimentally by optimizing the parameters of the laser pulse.

This revival of electronic coherence is interesting because the pump pulse is already off. This implies that the wave packet oscillates in the $B$ state and then goes back to the FC region, where it is still coherent with the part left in the ground state. To understand this more deeply we have analyzed the nuclear density function, Eq. (5). Results are illustrated in Fig. 4, with snapshots from the structure of the nuclear wave-packet density $\left|\Psi_{\text {nuc }}^{i}\left(R_{1}, R_{2}, t\right)\right|^{2}$ at different times. It is
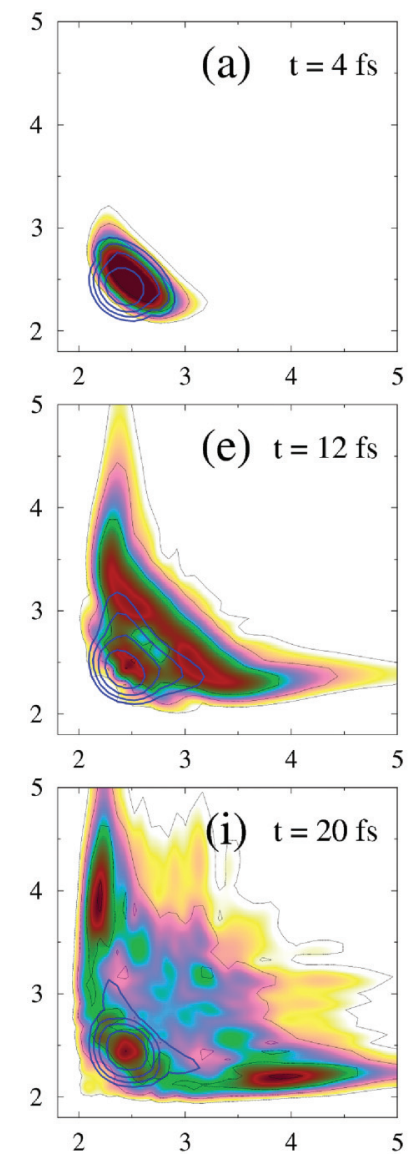
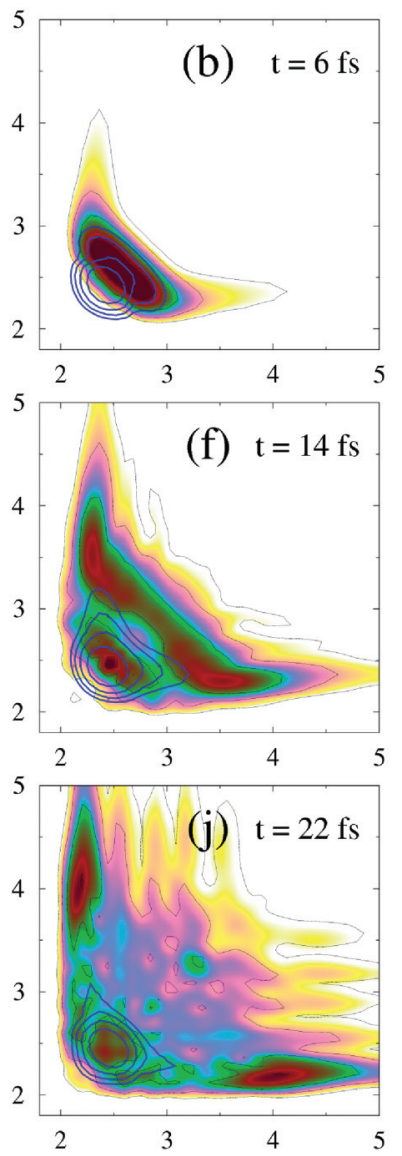
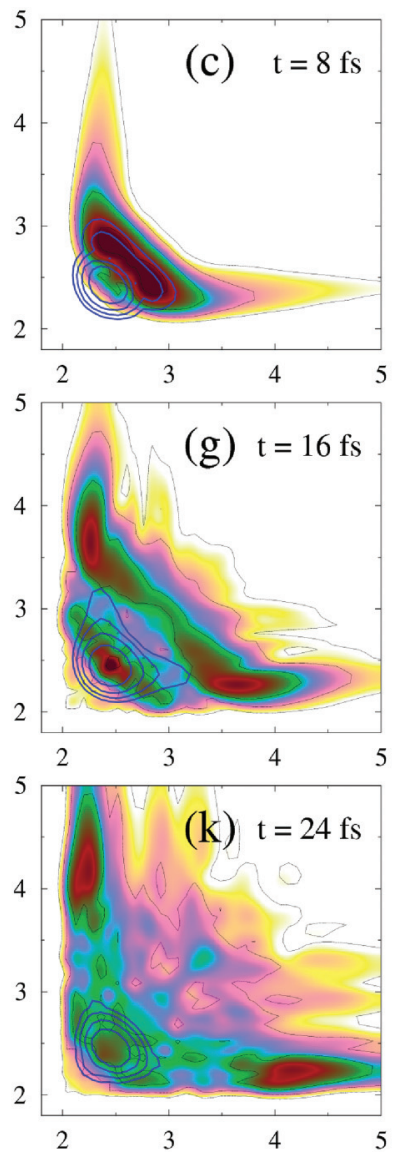
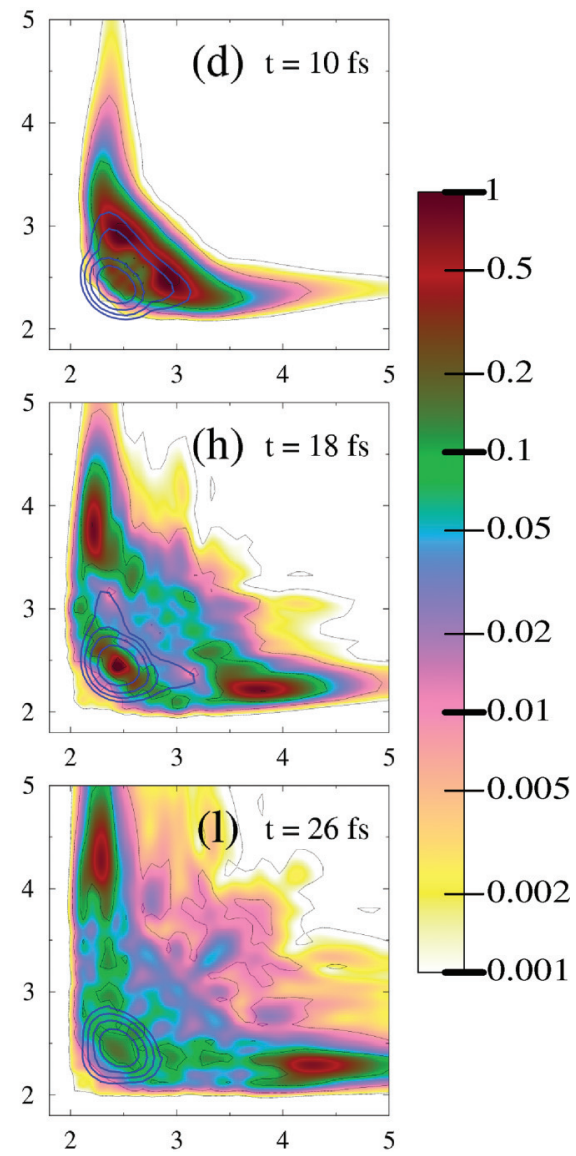

FIG. 4. (Color online) Snapshots of the time evolution of the nuclear wave-packet density along both O-O bonds (a.u.). 


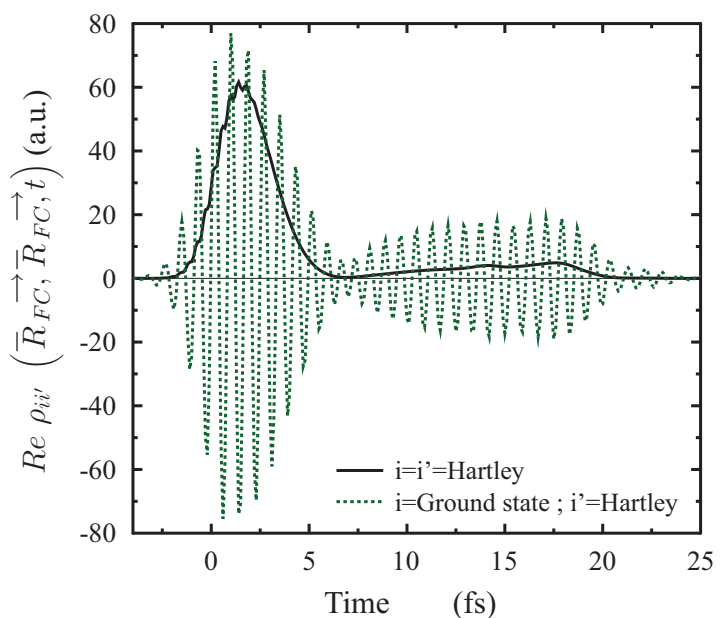

FIG. 5. (Color online) Local population density for state $B$ [solid (black) curve] and real part of the interference (last) term in Eq. (15) [dashed (green) curve] at the FC point as functions of time.

seen that a part of the nuclear wave packet stays trapped on the symmetric ridge of the $B \mathrm{PES}$, where both $\mathrm{O}-\mathrm{O}$ bonds increase synchronously. A valley-ridge inflection point occurs, where the nuclear wave packet splits into three components. One part is bound to come back to the FC region, while the rest dissociates along either of both equivalent channels.

The local population of the Hartley $B$ state at the FC point (see Fig. 5) has also been computed. We are, again, in the same situation as in Ref. [30], namely, state $B$ is populated significantly only during the first $\sim 5$-fs time interval over which the molecule remains around the FC region (at least approximately). However, in this case one part of the nuclear wave packet returns here again later.

The electronic wave packet is represented in Fig. 6 by the corresponding excited-state differential charge density at the FC point, Eq. (15). This local picture with respect to the nuclear coordinates illustrates the electron dynamics of the molecule at early times (during 5-6 fs), as long as the nuclear wave packets remain in the vicinity of the FC point.

The time-dependent coefficients are the values of both nuclear wave packets at the FC point obtained from MCTDH simulations (see also Fig. 5 for the corresponding local population of $B$ and coherence between $B$ and $X$ ). The charge and transition densities were obtained from electronic wave functions calculated at the SA-3-CAS $(18,12) /$ STO-3G level of theory using a development version of the Gaussian program [41]. We observed no qualitative difference in these when increasing the basis set to aug-cc-pVQZ or when adding a dynamic electron correlation at the MRCI level of theory using the MOLPRO program [40].

We see in Fig. 6 an oscillation of the electronic charge density from one bond to another with a period of $0.8 \mathrm{fs}$. The resulting electronic wave packet is thus an alternating superposition of two resonant forms, $\{\mathrm{O}--\mathrm{O}=\mathrm{O} \leftrightarrow \mathrm{O}=$ $\mathrm{O}$ - - $\mathrm{O}\}$, each having an excess or lack of electron on one or the other bond. These chemical structures are precursors of the two equivalent dissociation channels to $\mathrm{O}+\mathrm{O}_{2}$ or $\mathrm{O}_{2}+\mathrm{O}$. In addition, the revival of coherence is a signature that a part of the wave packet is trapped on the symmetrical ridge of the $B$-state PES, where it can overlap significantly with what is left in the ground state around the equilibrium geometry (see Fig. 4). This, and the subfemtosecond oscillation between both predissociating structures, suggests that the dissociation of ozone could perhaps be controlled by modulating the electron density on the attosecond time scale by involving a third electronic state coherently coupled to the $B$ state with a second pump pulse.

Finally, we must note here that the presented results can be applied straightforwardly only for initially aligned molecules. If this is not the case, one should take into consideration some additional effects. The individual molecules will feel a reduced intensity depending on their actual orientation. According to our test calculations the population of the $B$ state is nearly proportional to the effective intensity, while the relative coherence, Eq. (8), is nearly independent of it. This implies that, e.g., in the case of an isotropic molecular distribution (see Fig. 2), the population of state $B$ will be reduced by a factor of approximately 3 . Some similar change could be expected in Fig. 4. On the other hand, Figs. 3 and 5 remain valid even for nonaligned molecules. In the case of nonaligned molecules the most significant problem appears in the case of the presented time evolution of the excited differential electronic charge density (see in Fig. 6). For this situation it is only applicable if the probe process is appropriate to recover the actual orientation of the molecules.

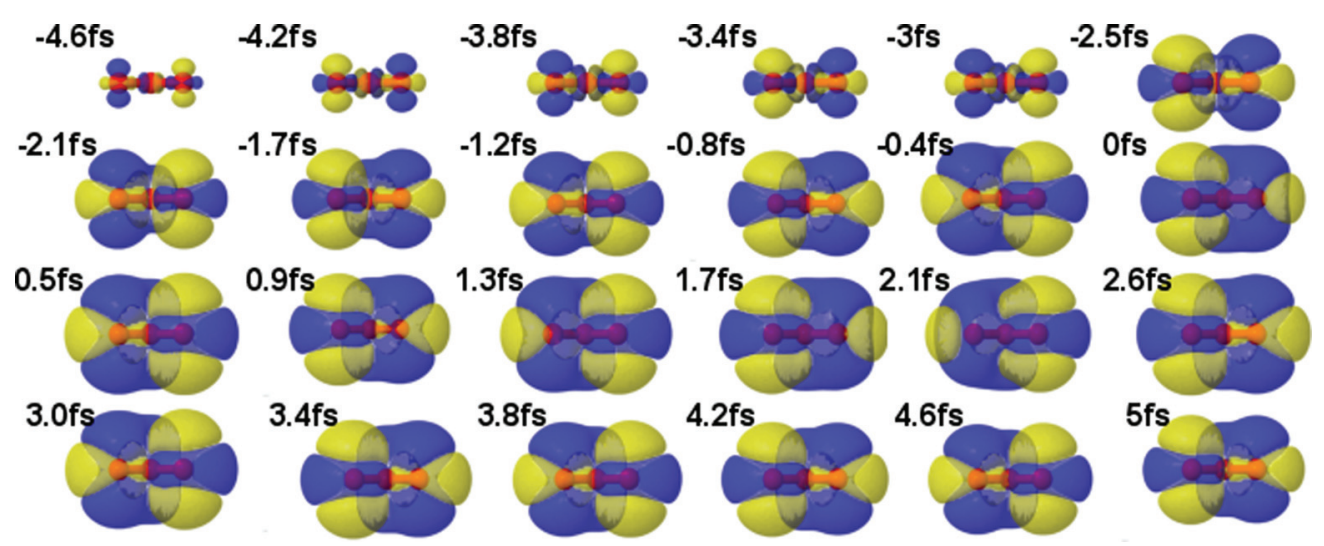

FIG. 6. (Color online) Time evolution of the excited differential electronic charge density, Eq. (15), at the FC geometry (side view). Dark (blue) area, hole; light (yellow) area, electron. 


\section{CONCLUSIONS}

In summary, we have performed numerical simulations of the coupled electron and nuclear motion in the ozone molecule on the attosecond time scale. An initial coherent nonstationary state was created as a coherent superposition of the ground and excited Hartley $B$ states. The MCTDH approach was applied to solve the dynamical Schrödinger equation for the nuclei in the framework of the time-dependent adiabatic partition including the light-matter interaction (electric dipole approximation).

A reasonably large electronic coherence has been obtained between the ground and the Hartley $B$ states during a short, 5-fs time interval. However, after this time an interesting phenomenon emerges. After the coherence decays within a certain period of time, a few femtoseconds later, it appears again. Nuclear wave-packet calculations support that we are presently in a situation where bifurcating reaction paths and valley-ridge inflection points are explored on the excited-state PES. The electronic motion during the first 5-6 fs shows an oscillation of the electronic charge density from one bond to another with a period of $0.8 \mathrm{fs}$. It is to be expected that this motion can be probed experimentally with an attosecond XUV pulse.

\section{ACKNOWLEDGMENTS}

The authors would like to thank F. Krausz, R. Kienberger, and M. Jobst for support and for fruitful discussions. We acknowledge R. Schinke for providing the potential energy surfaces and the transition dipole moment and H.-D. Meyer for fruitful discussions. The authors also acknowledge the TÁMOP 4.2.2.C-11/1/KONV-2012-0001 project. Á.V. acknowledges the OTKA (Grant No. NN103251). Financial support by the CNRS-MTA is gratefully acknowledged.

\section{APPENDIX}

Starting from Eq. (6) and performing further integration over the coordinates of the "last" electron and over the coordinates of the nuclei leads to

$$
\begin{aligned}
& \underbrace{\int_{(\vec{r})} \int_{(\vec{R})} \rho^{\mathrm{tot}}(\vec{r}, t, \vec{R}) d \tau d V}_{=1}=\underbrace{\int_{(\vec{R})}\left|\Psi_{\mathrm{nuc}}^{X}(\vec{R}, t)\right|^{2} d V}_{P^{X}(t)} \underbrace{\int_{(\vec{r})} \rho^{X}(\vec{r} ; \vec{R}) d \tau}_{=1}+\underbrace{\int_{(\vec{R})}\left|\Psi_{\mathrm{nuc}}^{B}(\vec{R}, t)\right|^{2} d V}_{P^{B}(t)} \underbrace{\int_{(\vec{r})} \rho^{B}(\vec{r} ; \vec{R}) d \tau}_{=1} \\
& +2 \operatorname{Re} \underbrace{\int_{(\vec{R})} \Psi_{\mathrm{nuc}}^{X *}(\vec{R}, t) \Psi_{\mathrm{nuc}}^{B}(\vec{R}, t) d V}_{S^{X B}(t)} \underbrace{\int_{(\vec{r})} \gamma^{X B}(\vec{r} ; \vec{R}) d \tau}_{=0}
\end{aligned}
$$

where $P^{X}(t)$ and $P^{B}(t)$ are the populations of states $X$ and $B$, respectively, at time t. $S^{X B}(t)$, the overlap of the nuclear wave packets on states $X$ and $B$, is a measure of the global coherence between state $X$ and state $B$ for all geometries. This shows that the interference term (involving the coherence and the transition density) does not directly contribute to the probability of finding the molecule in a given state (it does contribute indirectly, though, by having an effect on the time evolution of the populations).

We note the following here. (i) At the FC point the symmetry point group is $C_{2 v}$. By construction, charge densities are $A_{1}$ (totally symmetric). However, because the $X$ and $B$ states have $A_{1}$ and $B_{2}$ symmetries, the transition density is $B_{2}$ (antisymmetric with respect to the $C_{2}$ axis and the left-right mirror plane). (ii) $\Delta \rho^{B}\left(\vec{r}, \vec{R}_{\mathrm{FC}}\right)>0$ means a gain of electron density, whereas $\Delta \rho^{B}\left(\vec{r}, \vec{R}_{\mathrm{FC}}\right)<0$ means a loss of electron density, i.e., a gain of hole density. (iii) The sign of $\gamma^{X B}\left(\vec{r} ; \vec{R}_{\mathrm{FC}}\right)$ can be positive (constructive interference) or negative (destructive interference). In practice, it is not well defined because the signs of the electronic states are arbitrary (in fact their phases, but they are chosen real-valued). However, this does not matter in practice, because this term has $B_{2}$ symmetry, and both terminal oxygen atoms are equivalent through permutation.
[1] P. B. Corkum and F. Krausz, Nat. Phys. 3, 381 (2007).

[2] E. Goulielmakis et al., Science 320, 1614 (2008).

[3] F. Krausz and M. Ivanov, Rev. Mod. Phys. 81, 163 (2009).

[4] O. Smirnova, Y. Mairesse, S. Patchkovskii, N. Dudovich, D. Villeneuve, P. Corkum, and M. Yu. Ivanov, Nature 460, 972 (2009).

[5] Y. Huismans et al., Science 331, 61 (2011).

[6] X. Zhou, P. Ranitovic, C. W. Hogle, J. H. D. Eland, H. C. Kapteyn, and M. M. Murnane, Nat. Phys. 8, 232 (2012).
[7] N. Rohringer, A. Gordon, and R. Santra, Phys. Rev. A 74, 043420 (2006).

[8] N. Rohringer and R. Santra, Phys. Rev. A 79, 053402 (2009).

[9] F. Kelkensberg et al., Phys. Rev. Lett. 103, 123005 (2009).

[10] E. Goulielmakis et al., Nat. Lett. 466, 739 (2010).

[11] F. Remacle, R. Kienberger, F. Krausz, and R. D. Levine, Chem. Phys. 338, 342 (2007).

[12] F. Remacle and R. D. Levine, Z. Phys. Chem. 221, 647 (2007). 
[13] F. Remacle, M. Nest, and R. D. Levine, Phys. Rev. Lett. 99, 183902 (2007).

[14] M. Nest, Chem. Phys. Lett. 472, 171 (2009).

[15] B. H. Muskatel, F. Remacle, and R. D. Levine, Phys. Scripta 80, 048101 (2009).

[16] F. Remacle and R. D. Levine, Phys. Rev. A 83, 013411 (2011).

[17] B. Mignolet, R. D. Levine, and F. Remacle, Phys. Rev. A 86, 053429 (2012).

[18] C. M. Oana and A. I. Krylova, J. Chem. Phys. 127, 234106 (2007).

[19] L. S. Cederbaum, J. Chem. Phys. 128, 124101 (2008).

[20] A. D. Bandrauk, S. Chelkowski, and H. S. Nguyen, Int. J. Quant. Chem. 100, 834 (2004).

[21] A. D. Bandrauk, S. Chelkowski, P. B. Corkum, J. Manz, and G. L. Yudin, J. Phys. B 42, 134001 (2009).

[22] F. Kelkensberg, W. Siu, J. F. Perez-Torres et al., Phys. Rev. Lett. 107, 043002 (2011).

[23] B. Fischer, M. Kremer, T. Pfeifer, B. Feuerstein, V. Sharma, U. Thumm, C. D. Schröter, R. Moshammer, and J. Ullrich, Phys. Rev. Lett. 105, 223001 (2010).

[24] J. F. Pérez-Torres, F. Morales, J. L. Sanz-Vicario, and F. Martín, Phys. Rev. A 80, 011402(R) (2009).

[25] G. Sansone, F. Kelkensberg, J. F. Pérez-Torres et al., Nature 465, 763 (2010).

[26] S. Gräfe, V. Engel, and M. Y. Ivanov, Phys. Rev. Lett. 101, 103001 (2008).

[27] P. von den Hoff, I. Znakovskaya, M. F. Kling, and R. de VivieRiedle, Chem. Phys. 366, 139 (2009).

[28] I. Znakovskaya, P. von den Hoff, S. Zherebtsov, A. Wirth, O. Herrwerth, M. J. J. Vrakking, R. de Vivie-Riedle, and M. F. Kling, Phys. Rev. Lett. 103, 103002 (2009).
[29] P. von den Hoff, R. Siemering, M. Kowalewski, and R. de VivieRiedle, IEEE J. Sel. Top. Quantum Electron. 18, 1 (2012).

[30] G. J. Halász, A. Perveaux, B. Lasorne, M. A. Robb, F. Gatti, and Á. Vibók, Phys. Rev. A 86, 043426 (2012).

[31] M. Baer, Beyond Born Oppenheimer: Electronic Non-Adiabatic Coupling Terms and Conical Intersections (Wiley, Hoboken, NJ, 2006).

[32] H.-D. Meyer, U. Manthe, and L. S. Cederbaum, Chem. Phys. Lett. 165, 73 (1990).

[33] U. Manthe, H.-D. Meyer, and L. S. Cederbaum, J. Chem. Phys. 97, 3199 (1992).

[34] M. H. Beck, A. Jäckle, G. A. Worth, and H.-D. Meyer, Phys. Rep. 324, 1 (2000); G. A. Worth et al., The MCTDH Package, Version 8.2 (2000), Version 8.3 (2002), Version 8.4 (2007), University of Heidelberg, Germany. See http://mctdh.uni-hd.de/.

[35] H.-D. Meyer, F. Gatti, and G. A. Worth (eds.), Multidimensional Quantum Dynamics: MCTDH Theory and Applications (WileyVCH, Weinheim, 2009).

[36] Z.-W. Qu, H. Zhu, S. Yu. Grebenshchikov, and R. Schinke, J. Chem. Phys. 123, 074305 (2005).

[37] E. Baloïtcha and G. G. Balint-Kurti, J. Chem. Phys. 123, 014306 (2005).

[38] S. Y. Grebenshchikov, Z.-W. Qu, H. Zhu, and R. Schinke, Phys. Chem. Chem Phys. 9, 2044 (2007).

[39] R. Schinke and G. C. McBane, J. Chem. Phys. 132, 044305 (2010).

[40] H.-J. Werner, P. J. Knowles, R. Lindth et al., MOLPRO, Version 2006, 1, a Package of Ab Initio Programs; see http://www.molpro.net.

[41] M. J. Frisch et al., Gaussian Development Version, Revision H.01 (Gaussian, Inc., Wallingford, CT, 2009). 